\begin{tabular}{|c|c|}
\hline Title & $\begin{array}{l}\text { Boundary integral equation approach based on a polynomial expansion of the current distribution to reconstruct the } \\
\text { current density profile in tokamak plasmas }\end{array}$ \\
\hline Author(s) & Itagaki, Masafumi; Y amaguchi, Satoki; Fukunaga, T akaaki \\
\hline Citation & $\begin{array}{l}\text { Nuclear Fusion, 45(3), 153-162 } \\
\text { https://doi.org/10.1088/0029-5515/45/3/001 }\end{array}$ \\
\hline Issue Date & 2005-03 \\
\hline Doc URL & http:/hdl.handle.net/2115/58434 \\
\hline Rights & $\begin{array}{l}\text { (C) } 2005 \text { IOP Publishing Ltd.T his is an author-created, un-copyedited version of an article accepted for publication in } \\
\text { Nuclear Fusion. The publisher is not responsible for any errors or omissions in this version of the manuscript or any } \\
\text { version derived from it. The V ersion of Record is available online at } 10.1088 / 0029-5515 / 45 / 3 / 001\end{array}$ \\
\hline Type & article (author version) \\
\hline File Information & Nucl. Fusion, 45, 153-162 (2005)_TXT-1.pdf \\
\hline
\end{tabular}

Instructions for use 


\section{Boundary Integral Equation Approach Based on Polynomial Expansion of Current Distribution to Reconstruct Current Density Profile in Tokamak Plasma}

Masafumi ITAGAKI, Satoki YAMAGUCHI, Takaaki FUKUNAGA

Graduate School of Engineering, Hokkaido University,

Kita 13, Nishi 8, Kita-ku, Sapporo 060-8628, JAPAN

Tel. +81-11-706-6659, Fax. +81-11-747-9366

E-mail: itagaki@qe.eng.hokudai.ac.jp

This paper contains

24 pages of text

and 9 figures. 


\begin{abstract}
A new approach has been proposed to reconstruct the current density profile in Tokamak plasma. The boundary-only integral equation derived from the Grad-Shafranov equation under the assumption of polynomial expansion of current density will have no unknowns except for the polynomial expansion coefficients, once the magnetic flux and its derivative have been given along the plasma boundary with the aid of Kurihara's Cauchy-condition surface method based on magnetic sensor data. In addition to the discretized form of the equation, some constraints are taken into account: the total plasma current, zero-current along the plasma boundary, a scalar relationship derived from the MHD equilibrium to connect the current density with the magnetic flux. It is also assumed that the poloidal field as a quantity closely related to the current density can be measured at a certain number of points inside the plasma. The whole set of the linear equations are solved using the singular value decomposition technique to determine the polynomial expansion coefficients. The validity of the present technique and the quality of the current density solution has been investigated through test calculations for some plasma configurations.
\end{abstract}

PACS numbers: $\quad 28.52 .-\mathrm{s}, 28.52 . A v ., 52.55 .-\mathrm{s}, 52.55 . \mathrm{Fa}$ 


\section{Introduction}

The boundary shape of a tokamak plasma can nowadays be smoothly identified from signals of the magnetic flux and the poloidal magnetic field, using an on-line computing methodology based on the 'filament current approximation [1]' or the 'Cauchy-condition surface method [2]'. The next important step will be the reproduction of the spatial distribution of current density in the plasma domain whose boundary has been fixed by the above boundary-shape computation. This second process is to solve the Grad-Shafranov equation [3-6], which describes the MHD equilibrium in axisymmetric plasma like a tokamak, as a fixed boundary problem.

The most popular idea that has been widely used to reconstruct the internal current profile is to solve the Grad-Shafranov equation by iteratively adjusting a small number of free parameters characterizing the current density profile $j_{\varphi}$ (the toroidal component of the plasma current). These free parameters are chosen in such a way that the measured signals of magnetic sensors will be consistent with the results predicted by the calculation $[7,8]$.

In the present paper, the authors try to seek the plasma current profile in a different way; the above type of parameterization is not used in the present work. As the outline will be described in section 2, Itagaki et al. [9] derived a "boundary-only integral equation" by expanding the quantity $\mu_{0} r j_{\varphi}$ related to the plasma current profile into two-dimensional polynomial in terms of spatial coordinates $r$ and $z$. The most important feature in this formulation is that for numerical computations it requires discretization of the boundary only rather than of the plasma domain. This advantage is particularly important in a series of frequent analyses; geometry data generation and modifications are easily performed. That is, the method is well suited for on-line plasma equilibrium analysis that requires efficient data preparation and computation following the change in plasma shape during the operation of an actual fusion device. The authors believe that this boundary-only 
formulation shows its merit when applied to an 'inverse' problem to reproduce the plasma current profile from data fixed along the plasma boundary, as the reason for this will be suggested below.

Kurihara's Cauchy condition surface (CCS) method [2] was developed originally for the determination of the plasma boundary shape, however, it can also estimate values of the normal derivative $\partial \psi / \partial n$ as well as the magnetic flux function $\psi$ on the plasma boundary, as will be described in section 3 . This means, once the plasma boundary shape and the boundary conditions are fixed by the method, the above boundary-only integral equation has no unknowns any more except for the polynomial expansion coefficients. The coefficients and then the current density profile can be easily estimated, although one needs to add some constraints to successfully obtain a unique and stable solution. A priori information and physical constraints that will be taken into account in the present work are described in section 4. For example, in section 4.4, the authors propose a new method to estimate the current density from the raw measured data on poloidal field for a certain number of points in a line. All of these constraints can be described using the polynomial form, and then they can be incorporated into the discretized form of the boundary integral equation and then form a system of linear algebraic equations to determine the polynomial expansion coefficients. The detailed methodology based on the above procedure is also described in section 4 . Several numerical results are reported and discussed in section 5 and 6.

\section{Boundary-only integral equation for the Grad-Shafranov equation}

For an axisymmetric (r, z) system the Grad-Shafranov equation is given by

$$
-\Delta^{*} \psi \equiv-\left\{r \frac{\partial}{\partial r}\left(\frac{1}{r} \frac{\partial}{\partial r}\right)+\frac{\partial^{2}}{\partial z^{2}}\right\} \psi=\mu_{0} r^{2} \frac{d P}{d \psi}+\frac{d}{d \psi}\left(\frac{F^{2}}{2}\right) \equiv \mu_{0} r j_{\varphi},
$$

where the magnetic flux function $\psi$ is defined as $\psi=r A_{\varphi}$ with the toroidal component of vector 
potential $A_{\varphi}, j_{\varphi}$ denotes the toroidal component of the plasma current, $P$ the plasma pressure and $F$ the poloidal current function.

Itagaki et al [9] showed that the above Grad-Shafranov equation can be transformed into an equivalent boundary-only integral equation in terms of the plasma boundary $\Gamma$,

$$
c_{i} \psi_{i}-\int_{\Gamma}\left(\frac{\psi^{*}}{r} \frac{\partial \psi}{\partial n}-\frac{\psi}{r} \frac{\partial \psi^{*}}{\partial n}\right) d \Gamma=\sum_{l, m} \alpha_{l, m}\left\{c_{i} \varphi_{i}^{(l, m)}-\int_{\Gamma}\left(\frac{\psi^{*}}{r} \frac{\partial \varphi^{(l, m)}}{\partial n}-\frac{\varphi^{(l, m)}}{r} \frac{\partial \psi^{*}}{\partial n}\right) d \Gamma\right\},
$$

by assuming a polynomial expansion of the RHS of equation (1):

$$
\mu_{0} r j_{\varphi} \approx \sum_{l, m} \alpha_{l, m} \xi^{l} \eta^{m} . \quad(l \geq 0, m \geq 0)
$$

Here, $\xi$ and $\eta$ are dimensionless coordinates $\xi=r / L_{r}$ and $\eta=\left(z-z_{0}\right) / L_{z}$, respectively with appropriate constants $L_{r}, L_{z}$ and $z_{0}$. Note here that equation (3) itself does not include any information related to the equilibrium condition, $\mathbf{J} \times \mathbf{B}=\nabla p$, explicitly. In an actual analysis, one needs to add a restriction to consider this equilibrium condition, as will be shown in section 4.3.

In equation (2), $\psi^{*}$ denotes the fundamental solution which satisfies a subsidiary equation

$$
-\Delta^{*} \psi^{*}=r \delta_{i}
$$

where $\delta_{i}$ is Dirac's delta function with the spike at the point $i$. The detailed form of the fundamental solution is well known and found in literatures [10-12].

The constant $c_{i}$ in equation (2) depends on the local boundary geometry under consideration: $c_{i}=1.0$ for an internal point, while $c_{i}=1 / 2$ on a smooth boundary. The quantity $\varphi^{(l, m)}$ means a particular solution which satisfies the Grad-Shafranov equation with a monomial source:

$$
-\Delta^{*} \varphi^{(l, m)}=\xi^{l} \eta^{m}=\left(\frac{r}{L_{r}}\right)^{l}\left(\frac{z-z_{0}}{L_{z}}\right)^{m} \cdot \quad(l \geq 0, \quad m \geq 0)
$$

The detailed form of $\varphi^{(l, m)}$ is written as an infinite series [9]: 


$$
\varphi^{(l, m)}=-\frac{L_{z}^{2} \xi^{l} \eta^{m+2}}{(m+1)(m+2)}\left[1+\sum_{k=1}^{\infty} \prod_{s=1}^{k}\left\{-\frac{(l-2 s+2)(l-2 s)}{(m+2 s+1)(m+2 s+2)}\left(\frac{L_{z} \eta}{L_{r} \xi}\right)^{2}\right\} .\right.
$$

Note that the singularity constant $C_{i}$, the fundamental solution $\psi^{*}$ and the particular solution $\varphi^{(l, m)}$ in equation (2) are all known quantities. In addition to them, the boundary values of $\psi$ and $\partial \psi / \partial n$ as well as the plasma boundary shape can be evaluated through the analysis using the CCS method with the magnetic sensor data, as the details will be described in section 3 . It should be emphasized that eventually, in this case, unknown quantities left in equation (2) are only the polynomial expansion coefficients $\alpha_{l, m}$.

\section{Outline of the Cauchy-condition surface method}

The Cauchy-condition surface (CCS) is defined as a surface where both the Dirichlet condition and the Neumann condition are unknown. The CCS method [2] is an inverse analytic technique to determine the plasma boundary shape.

Figure 1. Illustration of Cauchy-condition surface method

First, one locates a CCS inside the actual plasma region, as illustrated in figure 1. One here assumes mathematically that there is no plasma, i.e., vacuum everywhere outside the CCS. Now, to evaluate the Dirichlet $(\psi)$ and the Neumann $(\partial \psi / \partial n)$ conditions at several points along the CCS ( $\Gamma_{C C S}$ ), three types of boundary integral equations for the vacuum field can be given using the sensor signals and the poloidal coil current data, as shown below.

(i) For the magnetic 'flux' signal $\psi_{i}$ at points $i$ :

$$
\int_{\Gamma_{C C S}}\left(\frac{\psi^{*}}{r} \frac{\partial \psi}{\partial n}-\frac{\psi}{r} \frac{\partial \psi^{*}}{\partial n}\right) d \Gamma=\psi_{i}-W_{i}^{\psi}
$$

where $W_{i}^{\psi}$ is the contribution of all poloidal coils to the point $i$, i.e., 


$$
W_{i}^{\psi}=\mu_{0} \sum_{k \in \text { Coils }} I_{k} \psi^{*}\left(\mathbf{r}_{k} \rightarrow \mathbf{r}_{i}\right)
$$

with $I_{k}$ being the electric current of the k-th coil.

(ii) For the magnetic 'field' signal $B_{i}$ located at points $i$ :

$$
\int_{\Gamma_{C C S}}\left(\frac{B^{*}}{r} \frac{\partial \psi}{\partial n}-\frac{\psi}{r} \frac{\partial B^{*}}{\partial n}\right) d \Gamma=B_{i}+\mathbf{n}_{0} \cdot \nabla W_{i}^{\psi} / r_{i} .
$$

where $B_{i}=-\mathbf{n}_{0} \cdot \frac{\nabla \psi_{i}}{r_{i}}, B^{*}=-\mathbf{n}_{0} \cdot \frac{\nabla \psi^{*}}{r}$ with $\mathbf{n}_{0}$ being the assigned vector normal to the direction of the 'magnetic probe' located at the point $i$.

(iii) For points $i$ along the Cauchy condition surface:

$$
\int_{\Gamma_{C C S}}\left(\frac{\psi^{*}}{r} \frac{\partial \psi}{\partial n}-\frac{\psi}{r} \frac{\partial \psi^{*}}{\partial n}\right) d \Gamma=\frac{1}{2} \psi_{i}-W_{i}^{\psi} .
$$

Equations (7a), (7b) and (7c) are discretized, coupled and can be expressed in a matrix form. Once all the values of $\partial \psi / \partial n$ and $\psi$ along the CCS have been given by solving the matrix equation in a least square sense, the distribution of magnetic flux $\psi_{i}$ can be calculated using equation (7a) for arbitrary points ' $i$ '. Thus, the outermost magnetic flux surface can be found by drawing contours of magnetic flux. Differential values of magnetic flux and so the poloidal field along the boundary can be also computed by differentiating the portions related to the fundamental solution in equation (7a).

\section{Present approach to reconstruct plasma current profile}

\subsection{Outline}

A discretized form of equation (2) is applied to a number of node points located along the plasma boundary, then, one will have a simultaneous linear equation. However, the equation is not sufficient to provide reliable solutions for $\alpha_{l, m}$. To avoid the ill-condition of the problem, one needs to add $a$ priori information and physical constraints into the above simultaneous linear equation. The whole 
set of the linear equations are solved using the singular value decomposition (SVD) technique [13] to determine $\alpha_{l, m}$. Once $\alpha_{l, m}$ have been determined, the profile of magnetic flux can be given by solving equation (2) directly for $\psi_{i}$ at points ' $i$ ' located inside the plasma.

\subsection{A priori information and physical constraints}

A priori information and physical constraints taken into account in the present work are as follows.

(1) The total plasma current is known.

(2) Zero-current condition is prescribed for each point along the plasma boundary.

(3) Constraints derived from the equilibrium $\mathbf{J} \times \mathbf{B}=\nabla p$ are imposed for points distributed in the plasma domain. In more detail, one adopts the scalar relationships described in section 4.3 to connect the current density with the magnetic flux or field.

(4) Assume that the poloidal field can be measured at a certain number of points along a straight line inside the plasma (see section 4.4).

\subsection{Detailed constraints derived from the equilibrium condition}

Kurihara transformed the equilibrium condition $\mathbf{J} \times \mathbf{B}=\nabla p$ into a scalar relationship between the plasma current and the poloidal field [14],

$$
\frac{r^{3}}{B_{r}}\left\{B_{r} \frac{\partial}{\partial r}\left(\frac{j_{\varphi}}{r}\right)+B_{z} \frac{\partial}{\partial z}\left(\frac{j_{\varphi}}{r}\right)\right\}=-\frac{1}{\mu_{0}} \frac{d}{d \psi}\left(r B_{\varphi}\right)^{2},
$$

by incorporating the Maxwell equation $\mu_{0} \mathbf{J}=\nabla \times \mathbf{B}$ and $\nabla \cdot \mathbf{B}=0$. In equation (8), $B_{r}$ and $B_{z}$ are the poloidal magnetic field, i.e., $B_{r}=(-1 / r)(\partial \psi / \partial z)$ and $B_{z}=(1 / r)(\partial \psi / \partial r)$, respectively. The quantity $B_{\varphi}$ means the toroidal magnetic field, and it should be noted that $r B_{\varphi}$ is a magnetic surface function. One here simplifies equation (8) for the convenience to handle the polynomial expansion of $\mu_{0} r j_{\varphi}$, as, 


$$
r \frac{\partial}{\partial r} \sum_{l, m} \alpha_{l, m} \xi^{l} \eta^{m}+r \frac{B_{z}}{B_{r}} \frac{\partial}{\partial z} \sum_{l, m} \alpha_{l, m} \xi^{l} \eta^{m}-2 \sum_{l, m} \alpha_{l, m} \xi^{l} \eta^{m} \equiv-\frac{d}{d \psi}\left(r B_{\varphi}\right)^{2}
$$

For points $B_{r}=0$ or in the neighborhood the second term on the LHS should be replaced by

$$
r \frac{B_{z}}{\partial B_{r} / \partial z} \frac{\partial^{2}}{\partial z^{2}} \sum_{l, m} \alpha_{l, m} \xi^{l} \eta^{m}
$$

using L'hopital's rule to avoid numerical instability. The quantity $-d\left(r B_{\varphi}\right)^{2} / d \psi$ is also a magnetic surface function. This means that the LHS of equation (9) and the magnetic flux have the same contour. A subroutine drawing contours of magnetic flux was introduced into the authors' equilibrium program. Eight points are set along each magnetic surface. Then one applies the condition that any two points have the same value determined by equation (9). Up to ${ }_{8} C_{2}=28$ pairs of equilibrium conditions can be imposed, as shown in figure 2 .

Figure 2. Twenty-eight pairs of equilibrium conditions on a magnetic surface

One can also introduce another constraint:

$$
\frac{\partial}{\partial z} \sum_{l, m} \alpha_{l, m} \xi^{l} \eta^{m}=0 \quad \text { for points } \quad B_{r}=-\frac{1}{r} \frac{\partial \psi}{\partial z}=0
$$

Equation (10) can be easily derived if one finds

$$
\frac{\partial}{\partial z}\left(\mu_{0} r j_{\varphi}\right)=\left\{\mu_{0} r^{2} \frac{d^{2} P}{d \psi^{2}}+\frac{d^{2}}{d \psi^{2}}\left(\frac{F^{2}}{2}\right)\right\} \frac{\partial \psi}{\partial z}=0 .
$$

Here one must note that the above two types of constraints require 'iterations' to solve alternatively the 'unknown' magnetic flux profile from the current density distribution. Usually the not-converged current density solution given at an early stage of iteration looks "dirty”, as will be shown in section 5, however, the quality of the flux profile calculated using even this dirty current density does not tend to be very bad. This can be understood from the mathematical form of the Grad-Shafranov equation. Thus the applications of equations (9) and (10) to the calculated magnetic flux are successful in a practical sense. 


\subsection{Current density estimated from measured poloidal field}

As some researchers $[10,15,16]$ already pointed out, information obtained only from external magnetic measurements is insufficient for reliable evaluation of plasma current profile. One possible restriction to ensure a reliable reconstruction is to impose measured values of current density or a closely related physical quantity for a certain number of points inside the plasma. For test calculations in section 5, the authors assume that the poloidal field $B_{p}$ can be measured along a line in r-direction. In fact, it is known that the magnetic field pitch angle $\gamma_{p}=\tan ^{-1}\left(B_{p} / B_{\varphi}\right)$ can be evaluated in the motional Stark effect (MSE) measurement [17].

The authors use a method similar to that described in the literature [18] to estimate the current density from the raw measured data on poloidal field. As will be shown in section 5 , the calculated magnetic surfaces in an inverse equilibrium analysis are almost similar to the true ones even when the iteration is not yet converged. Due to this fact, one here assumes that the ratio of the poloidal field components obtained in the inverse analysis, say, $B_{r}^{I n v}$ and $B_{z}^{I n v}$, represents almost the true value. One then normalizes each of them using the measured poloidal field value $B_{p}^{M}$ for point $(r, z)$ on a straight line in r-directon, as,

$$
\begin{aligned}
& B_{r}(r, z)=B_{r}^{I n v}(r, z)\left\{B_{p}^{M}(r, z) / B_{p}^{I n v}(r, z)\right\}, \\
& B_{z}(r, z)=B_{z}^{I n v}(r, z)\left\{B_{p}^{M}(r, z) / B_{p}^{I n v}(r, z)\right\} .
\end{aligned}
$$

In the same way, one also obtains normalized values of $B_{r}$ and $B_{z}$ for points $(r+\Delta r, z)$ and $(r-\Delta r, z)$ using interpolated values of $B_{p}^{M}$. The quantity $\partial B_{z} / \partial r$ is calculated as

$$
\frac{\partial B_{z}}{\partial r} \approx \frac{B_{z}(r+\Delta r, z)-B_{z}(r-\Delta r, z)}{2 \Delta r}
$$

in a difference approximation. Next, one calculates 


$$
\begin{aligned}
\Delta B_{z} & =B_{z}(r, z+\Delta z)-B_{z}(r, z-\Delta z) \\
& =-\left\{B_{r}(r+\Delta r, z)-B_{r}(r-\Delta r, z)\right\} \frac{\Delta z}{\Delta r}-B_{r}(r, z) \frac{2 \Delta z}{r}
\end{aligned}
$$

from $\nabla \cdot \mathbf{B}=0$, i.e., $\partial B_{z} / \partial z+\partial B_{r} / \partial r+B_{r} / r=0$. Now one assumes simply

$$
\begin{aligned}
& B_{z}(r, z+\Delta z)=B_{z}(r, z)+\Delta B_{z} / 2, \\
& B_{z}(r, z-\Delta z)=B_{z}(r, z)-\Delta B_{z} / 2 .
\end{aligned}
$$

The values of $B_{r}$ in z-direction (where no measured data is given) can then be given by

$$
\begin{aligned}
& B_{r}(r, z+\Delta z)=B_{z}(r, z+\Delta z)\left\{\frac{B_{r}^{I n v}(r, z+\Delta z)}{B_{z}^{I n v}(r, z+\Delta z)}\right\}, \\
& B_{r}(r, z-\Delta z)=B_{z}(r, z-\Delta z)\left\{\frac{B_{r}^{I n v}(r, z-\Delta z)}{B_{z}^{I n v}(r, z-\Delta z)}\right\} .
\end{aligned}
$$

under the assumption that the ratio $B_{r} / B_{z}$ is preserved. Using these quantities one calculates

$$
\frac{\partial B_{r}}{\partial z} \approx \frac{B_{r}(r, z+\Delta z)-B_{r}(r, z-\Delta z)}{2 \Delta z} .
$$

Substituting equations (11) and (12) into the equation derived from Ampere’s law,

$$
j_{\varphi}=\left(\partial B_{r} / \partial z-\partial B_{z} / \partial r\right) / \mu_{0},
$$

one finally obtains the current density at the point $(r, z)$. Values of $j_{\varphi}$ thus calculated will be imposed as constraints at some points in a line.

\subsection{Whole matrix equation to determine polynomial expansion coefficients}

Conveniently enough, the above various constraints can be all expressed using the polynomial expansion approximation given by equation (3). The constraints are now rewritten as follows.

(i) From the boundary integral equation (2) for each node point $i$ along the boundary, one has

$$
\sum_{l, m} Q_{i}^{(l, m)} \alpha_{l, m}=c_{i} \psi_{i}-\int_{\Gamma}\left(\frac{\psi^{*}}{r} \frac{\partial \psi}{\partial n}-\frac{\psi}{r} \frac{\partial \psi^{*}}{\partial n}\right) d \Gamma, \quad(i=1,2, \cdots, N)
$$

where 


$$
Q_{i}^{(l, m)}=c_{i} \varphi_{i}^{(l, m)}-\int_{\Gamma}\left(\frac{\psi^{*}}{r} \frac{\partial \varphi^{(l, m)}}{\partial n}-\frac{\varphi^{(l, m)}}{r} \frac{\partial \psi^{*}}{\partial n}\right) d \Gamma
$$

(ii) The total plasma current is defined as $I=\int_{\Omega} j_{\varphi} d \Omega$, then,

$$
\sum_{l, m}\left(\frac{1}{\mu_{0} L_{r}} \int_{\Omega} \xi^{l-1} \eta^{m} d \Omega\right) \alpha_{l, m}=I . \quad(l \geq 0, m \geq 0)
$$

As the domain integral $\int_{\Omega} \xi^{l} \eta^{m} d \Omega$ can be transformed into a boundary one, i.e.,

$$
\int_{\Omega} \xi^{l-1} \eta^{m} d \Omega=\int_{\Omega} \nabla^{2} \phi^{(l-1, m)} d \Omega=\int_{\Gamma} \frac{\partial \phi^{(l-1, m)}}{\partial n} d \Gamma
$$

each value of the integral can be easily computed. The particular solution to $\nabla^{2} \phi^{(l-1, m)}=\xi^{l-1} \eta^{m}$ for $l \geq 1$ and $m \geq 0$ is shown in the literature [9]. As a special case, one also finds

$$
\phi^{(-1, m)}=L_{r}^{2}\left(\ln \xi+\ln L_{r}-1\right) \xi \eta^{m}+\sum_{s=1}^{\left[\frac{m}{2}\right]} c_{s}\left(\ln \xi+\ln L_{r}-1-d_{s}\right) \xi^{2 s+1} \eta^{m-2 s}
$$

with coefficients $c_{s}$ and $d_{s}$ that are calculated recurrently.

(iii) The value of $\mu_{0} r j_{\varphi}$ at each node $i$ along the plasma boundary is zero, then,

$$
\sum_{l, m}\left(\xi_{i}^{l} \eta_{i}^{m}\right) \alpha_{l, m}=0 . \quad(i=1,2, \cdots, N)
$$

(iv) If one assumes that at internal points $k$ the values of current density, $j_{\varphi, k}$, can be estimated through the procedure shown in section 4.4, one has

$$
\sum_{l, m}\left(\xi_{k}^{l} \eta_{k}^{m}\right) \alpha_{l, m}=\mu_{0} r j_{\varphi, k} \cdot \quad(k=1,2, \cdots, K)
$$

(v) Derivative of $\mu_{0} r j_{\varphi}$ in terms of $z$ takes the value of zero at points $p$ along the line $B_{r}=0 \quad$ (see equation (10)), one has

$$
\sum_{l, m}\left(m \xi_{p}^{l} \eta_{p}^{m-1}\right) \alpha_{l, m}=0 \quad(p=1,2, \cdots, P)
$$

(vi) From the equilibrium condition given by equation (9), one can derive a relationship between two 
points $2 q-1$ and $2 q$ that have the same value of magnetic flux.,

$$
\sum_{l, m}\left(\frac{l}{L_{r}} \sigma_{q, r}+\frac{m}{L_{z}} \sigma_{q, z}-2 \sigma_{q, 0}\right) \alpha_{l, m}=0, \quad(q=1,2, \cdots, Q)
$$

where

$$
\begin{gathered}
\sigma_{q, r}=r_{2 q} \xi_{2 q}^{l-1} \eta_{2 q}^{m}-r_{2 q-1} \xi_{2 q-1}^{l-1} \eta_{2 q-1}^{m}, \\
\sigma_{q, z}=r_{2 q} \frac{B_{z, 2 q}}{B_{r, 2 q}} \xi_{2 q}^{l} \eta_{2 q}^{m-1}-r_{2 q-1} \frac{B_{z, 2 q-1}}{B_{r, 2 q-1}} \xi_{2 q-1}^{l} \eta_{2 q-1}^{m-1}
\end{gathered}
$$

and

$$
\sigma_{q, 0}=\xi_{2 q}^{l} \eta_{2 q}^{m}-\xi_{2 q-1}^{l} \eta_{2 q-1}^{m}
$$

For points $B_{r}=0$ or in the neighborhood, the above quantity $\sigma_{q, z}$ should be replaced by

$$
\sigma_{q, z}=\frac{m-1}{L_{z}}\left(\frac{r_{2 q} B_{z, 2 q}}{\partial B_{r, 2 q} / \partial z} \xi_{2 q}^{l} \eta_{2 q}^{m-2}-\frac{r_{2 q-1} B_{z, 2 q-1}}{\partial B_{r, 2 q-1} / \partial z} \xi_{2 q-1}^{l} \eta_{2 q-1}^{m-2}\right) .
$$

Equations (14) to (19) commonly have $\alpha_{l, m}$ as unknowns. These equations can be therefore unified, and then one has the whole matrix equation

$$
\mathbf{A \alpha}=\mathbf{f}
$$

with $\boldsymbol{\alpha}=\left\{\alpha_{00}, \alpha_{10}, \alpha_{01}, \cdots, \alpha_{l, m}, \cdots\right\}^{T}$, the unknown vector of polynomial expansion coefficients.

The total number of lines in the matrix $\mathbf{A}$ is $2 N+1+K+P+Q$, while the number of columns agrees with the number of terms in the polynomial.

Equation (20) is solved using the singular value decomposition (SVD) technique [13]. In this technique, the matrix $\mathbf{A}$ is decomposed as $\mathbf{A}=\mathbf{U} \mathbf{\Lambda} \mathbf{V}^{\mathbf{T}}$, where $\mathbf{U}$ and $\mathbf{V}^{\mathbf{T}}$ are orthogonal matrices and the symbol $\mathbf{T}$ denotes the matrix transpose, while $\boldsymbol{\Lambda}$ is a diagonal matrix with positive singular value or zero components. Basically the solution is given by $\boldsymbol{\alpha}=\mathbf{V} \boldsymbol{\Lambda}^{-1} \mathbf{U}^{\mathrm{T}} \mathbf{f}$, however, one can employ the Tikhonov regularization [19] to stabilize the numerical ill-posedness. 
In this case the stabilized solution is given as

$$
\boldsymbol{\alpha}=\mathbf{V}\left(\boldsymbol{\Lambda}^{\mathrm{T}} \boldsymbol{\Lambda}+\gamma \mathbf{I}\right)^{-1} \boldsymbol{\Lambda}^{\mathrm{T}} \mathbf{U}^{\mathrm{T}} \mathbf{f}
$$

using the Tikhonov regularization parameter $\gamma$.

\section{Numerical demonstration for a parabolic current profile}

One here considers a problem to model the JT-60 tokamak-device. By the courtesy of Japan Atomic Energy Research Institute (JAERI), the reference data of plasma boundary, distributions of plasma current density and magnetic flux were firstly provided, which had been obtained from a 'forward' analysis using a reliable equilibrium code, SELENE [20]. This equilibrium computation was made based on a "parabolic" current profile parametrization that has the form

$$
\mu_{0} r j_{\varphi}=c_{0}\left\{\beta_{p} r^{2}+\left(1-\beta_{p}\right) R_{0}^{2}\right\}(1-X)^{0.6}
$$

Here, $X=\left(\psi-\psi_{M}\right) /\left(\psi_{S}-\psi_{M}\right)$ in which $\psi_{M}$ and $\psi_{S}$ are the values of $\psi$ on the magnetic axis and on the boundary, while $\beta_{p}(=0.70)$ and $R_{0}(=3.50 \mathrm{~m})$ denote the poloidal beta and the characteristic major radius, respectively. Data of poloidal coil currents and the signals of magnetic sensors (15 flux loops and 20 tangential probes) were also provided from the above SELENE calculation, and they can be used as input conditions for the following inverse analysis based on the CCS method to give both the plasma boundary shape and the boundary conditions.

\subsection{Plasma boundary calculation using the CCS method}

In the CCS computing code that was made in Hokkaido University, 3 quadratic boundary elements [21] and then 6 nodal points are used to model the Cauchy condition surface.

Solid lines in figure 3 show the contours of the magnetic flux obtained using the CCS method, while the dotted line denotes the plasma boundary evaluated by the SELENE code. The profiles of 
magnetic flux drawn inside the outermost magnetic flux surface, obtained using the CCS method, have no physical meaning. Nevertheless, the outermost magnetic flux surface agrees quite well with the reference boundary shape calculated by the SELENE code.

Figure 3. Plasma boundary shape reconstructed using the CCS method

Figure 4 compares the values of poloidal field $B_{p}=(1 / r) \partial \psi / \partial n$ along the plasma boundary. The abscissa axis denotes the distance from the X-point in counterclockwise direction along the boundary. The dotted line shows $B_{p}$ obtained from the 'direct' analysis with the boundary element method (BEM) based on equation (2), while the solid line shows $B_{p}$ calculated using the CCS method. The plasma boundary shape and the boundary values of $\partial \psi / \partial n$ thus obtained were used as input conditions for the following reconstruction of current density profile.

Figure 4. Comparison of boundary values of poloidal field calculated using the CCS method with the direct BEM solutions

The total plasma current $I$ can be also obtained utilizing the above CCS calculation results and performing the boundary integral along the plasma boundary $\Gamma$, i.e.,

$$
I=\frac{1}{\mu_{0}} \int_{\Gamma} \frac{1}{r} \frac{\partial \psi}{\partial n} d \Gamma
$$

For this JT-60 problem, in which the total current (correct answer) is $1.440 \mathrm{MA}$, the value numerically calculated using equation (23) is $1.438 \mathrm{MA}$ (the relative error is under $0.2 \%$ ). This suggests that the measurement of the total plasma current would be unnecessary in some situations (see also section 5.5).

\subsection{Reconstructed profiles of current density and magnetic flux in plasma}

To discretize the boundary integral equation (2), the plasma boundary was approximated by a polygon that has 80 sides, i.e., a total of 80 constant boundary elements [9, 21, 22] were employed. A 
8-th order complete polynomial was adopted to approximate $\mu_{0} r j_{\varphi}$, and hence the polynomial consists of a total of 45 unknown coefficients. The constraints (1) to (4) listed in section 4.2 were added to the above boundary integral information to assemble the whole matrix equation. The total plasma current was set to be $1.440 \mathrm{MA}$, the same value that was used for the SELENE forward analysis. Also, the condition of zero magnetic flux was prescribed along the plasma boundary. The Tikhonov regularization parameter is set to be $\gamma=10^{-9}$ for every iteration stage in the analysis.

The calculation results at the initial stage are show in figure 5. In figures 5(a) and 5(b), the solid lines represent the present inverse analysis results, while the dotted lines show the SELENE reference profiles. Since any equilibrium condition cannot be imposed at this initial stage, the current density distribution in figure 5(a) is badly distorted. However, as shown in figure 5(b), the magnetic flux surfaces calculated using this current density distribution are almost similar to the reference profiles. In figure 5(b), 8 points illustrated as "EQU points" were automatically set on each contour of magnetic flux drawn in every $0.1[\mathrm{~Wb}]$, so that for the second and the further iterations the equilibrium condition given by equation (19) is applied to $28\left({ }_{8} C_{2}\right)$ pairs along each contour. To impose the other equilibrium condition given by equation (18), 30 points were located along the line $B_{r}=0$ shown in this figure with the magnetic axis.

One assumes that the poloidal field ( $\left.B_{p}\right)$ is measured along the line $z=0$. Figure 5(c) shows the measured $B_{p}$ by the fine dashed curve, the calculated $B_{p}$ by the solid curve, and the ratio of these quantities by the dot-dashed one. The bold dashed curve shows the reference of parabolic current density, while the fine dotted curve denotes the current density calculated directly using equation (13) without modifying the calculated $B_{r}$ and $B_{z}$. Twenty current densities estimated according to the procedure described in section 4.4 are also plotted. These "modified" values are relatively close to the reference current density curve. However, some points show inaccurate values, 
which are caused by the drastic variation in the $B_{p}$ ratio near the magnetic axis. Because of this, three points are not adopted as the constraints in the following iteration.

Figure 5. Reconstructed results for a parabolic current profile (the initial stage)

The qualities of calculated results have been dramatically improved in the 5th iteration. In figure 6(a) and 6(b), 17 points out of 20 along the line $z=0$ are plotted, where current densities were estimated from the measured poloidal fields. Owing to the restrictions of these estimated current density values and also the equilibrium conditions, the current density profile shown in figure 6(a) agrees quite well with the reference. Each contour of magnetic flux (figure 6(b)) shows an excellent agreement with the reference.

The calculated curve of $B_{p}$ have become considerably close to the measured one, as shown in figure 6(c). However, due to the relatively large differences for small values of $B_{p}$, the $B_{p}$ ratio still represents a drastic change near the magnetic axis. Consequently the three estimated current densities in the center part are still far from the true values, although other points are relatively well approximated. The current density curve denoted by the fine dotted line, which was calculated without using the measured $B_{p}$, has also become quite close to the reference.

Figure 6. Reconstructed results for a parabolic current profile (the 5th iteration)

\subsection{Residual errors of the matrix equation}

Now one compares the original right-hand side vector of matrix equation (20) with the result of (matrix)*(solution vector) in the 5th iteration. The relative residual errors of equation (14), a subset of equation (20), which is related to 80 boundary integral equations, are less than $1.5 \%$ but $0.7 \%$ in average. For equation (15), the relative residual error of total current (1.429MA to the reference $1.440 \mathrm{MA}$ ) is $0.7 \%$. The average error of the 17 estimated current densities in equation (17) is $3.5 \%$, 
but the maximum error reaches $19.3 \%$. For equations (16), (18) and (19), which describe zero current along the boundary (80 points), the condition for the line $B_{r}=0$ (30 points) and the equilibrium conditions of 28pairs on each of 6 magnetic surface, the root mean square deviations from the zero values are $3.4 \times 10^{-2}, 2.4 \times 10^{-2}$ and $2.6 \times 10^{-2}$, respectively.

\subsection{The effect of measurement errors}

The effect of measurement errors on the reconstruction was also studied. Noises were numerically generated using normal (Gaussian) random numbers with a 3\% standard deviation and added to both the magnetic sensor signals and the measured poloidal field. Figure 7(a) shows the current density profile thus reconstructed in the 5th iteration, although other calculation conditions are the same as those in the above analysis. The reconstructed profile in the plasma center is obviously a little shifted to the left from the reference.

Next, each of the left 10 points and the right 10 points among the total of 20 noised poloidal field values were fit to a cubic polynomial in a least square sense, while such a smoothing was not applied to the noised magnetic sensor signals. The result in this case is shown in figure 7(b). Compared to figure 7(a), the quality of the profile has been well improved and considerably close to that in figure 6(a). It is also understood from this result that the errors of magnetic sensor signals are not very sensitive to the reconstruction since the CCS computation is made in a least square sense as described in section 3 .

Figure 7. Reconstructed current density profiles with noise effect

\subsection{The restriction on the total plasma current}

As another important matter, it should be reported here, the fact is, that the restriction on the total plasma current is unnecessary. There is no distinct difference between solutions reconstructed with and without this constraint. This is because the given information on the boundary conditions of 
$\partial \psi / \partial n$ are enough to specify the total current, as suggested at the end of section 5.1. The introduction of this constraint, however, never disturbs the reconstruction. Test calculations shown in this section were all performed with this restriction of total current.

\section{Some other test calculations}

The current density profile has been well reconstructed for parabolic plasma in section 5 . In this section, one deals with problems of a hollow current profile and an up-down symmetric configuration, both of which are more challenging than a parabolic profile.

\subsection{A hollow current profile}

The hollow current profile considered here is given by the parameterization

$$
\mu_{0} r j_{\varphi}=c_{0}\left\{\beta_{p} r^{2}+\left(1-\beta_{p}\right) R_{0}^{2}\right\}\left(1+3 X-4 X^{2}\right)
$$

with $\beta_{p}=0.70199, R_{0}=3.39927$ and $X$ being the normalized flux defined at the beginning of section 5. Following the CCS analysis for sensor signals given from a SELENE reference calculation, the boundary shape and boundary conditions were given in the same way described in section 5.1. The current profile reconstruction was made also using the same conditions and procedure shown in section 5.2, except that the Tikhonov regularization parameter was set to be $\gamma=10^{-8}$ and the flux contours were drawn every $0.07[\mathrm{~Wb}]$ to apply the equilibrium conditions for enough number of points.

The converged solutions obtained in the 5th iteration are shown in figure 8. One cannot say that the reconstructed profiles shown in figure 8(a) and 8(b) agree well with the reference data, compared to the results for parabolic plasma (see also figure 6). However, the reconstructed contours of current density in figure 8(a) do not intersect the reference ones, and it represents the characteristic of the hollow plasma as also suggested from the fine dotted curve in figure 8(c). 
Figure 8. Reconstructed results for a hollow current profile (the 5th iteration; $\gamma=10^{-8}$ )

\subsection{An up-down symmetric configuration}

One here considers circular plasma that has a radius of $1.0[\mathrm{~m}]$ with the center being $(r, z)=(3.5[\mathrm{~m}], 0.0[\mathrm{~m}])$ as a typical case of an up-down symmetry. One assumed in this case that the current profile obeys the parameterization for parabolic plasma given by equation (22). The plasma boundary conditions of $\partial \psi / \partial n$ were simply determined from a forward BEM calculation without using the CCS computation.

The drawback of the present method is that it cannot solve precisely a problem of up-down symmetry. One of the reasons is that some of the equilibrium pairs of condition given by equation (19) cause the matrix to be singular when applied to such geometry. Further, the set of equation (14) itself is very ill-conditioned for this up-down symmetry. However, the application of the Tikhonov regularization has an effect to stabilize the matrix singularity and ill-posedness. Figure 9(a) shows the current density profile obtained under the condition $\gamma=10^{-9}$. Most of the calculation conditions were the same as in section 5 , although this profile was given in the 10th iteration, as the convergence was a little slow. The reconstructed current density shows an up-down symmetry but the profile is slightly shifted to the left.

Figure 9(b) is the result obtained without applying the Tikhonov regularization but adopting the following "up-down symmetry option". The points on the lower part among 8 points on a magnetic surface were not used to apply the equilibrium condition (19). Instead, for 3 pairs in z-direction on each surface one applies a new condition that both point of each pair has the same current density. Because of no application of the regularization, the current profile in the early stages of iteration was severely violated, however, the profile was gradually improved. Figure 9(b) is the result in the 10th iteration. The quality of the profile is almost the same as in figure 9(a). In future the "method of images [23]" is worth trying to avoid the ill-posedness of the set of equation (14) caused by the 
up-down symmetry.

Figure 9. Circular current profiles reconstructed in the 10th iterations

\section{Conclusions}

A new approach to reconstruct the plasma current profile has been proposed, and the discussion was made about its validity. As a result, the following conclusions have been obtained:

1. The iteration to impose the equilibrium conditions converges well.

2. For a reliable reconstruction it is effective to introduce the current densities estimated from measured poloidal fields using the technique shown in section 4.4 to a certain number of points in the plasma interior.

3. The reconstructed result is quite accurate at least for parabolic plasma. However, more effort must be made to expand the application of the present method to various types of plasma current profiles.

4. According to the test calculations assuming random noise with a $3 \%$ standard deviation, the error caused by the magnetic sensor signal noise is almost negligible, while the noise on the measured poloidal field can be smoothed to obtain an acceptable quality of current profile, as shown in section 5.4 .

5. The restriction on the total plasma current is unnecessary

6. Guidelines need to be established for the best combination of analytic conditions as well as to seek the optimized value of the Tikhonov regularization parameter.

\section{Acknowledgements}

This paper includes the achievement of 'Fiscal 2003 Research Cooperation with JAERI on 
Experiments and Analyses Using the Critical Plasma Test Equipment JT-60’. The authors wish to express their gratitude to Dr. K. Kurihara of JAERI for his valuable and helpful comments on this work. Further, he kindly provided them with the reference tokamak plasma data used in the numerical demonstration in section 5 and 6 . The authors should mention here that the referees kindly gave them many suggestions to improve the method and the results.

\section{References}

(1) Swain, D.W., Neilson, G.H., Nucl. Fusion, 22 (1982) 1015.

(2) Kurihara, K., Fusion Eng. Des., 51-52 (2000) 1049.

(3) Shafranov, V.D., Sov. Phys. - JETP 37 (1960) 775.

(4) Mukhovatov, V.S., Shafranov, V.D., Nucl. Fusion 11 (1971) 605.

(5) Shafranov, V.D., Plasma Phys. 13 (1971) 757

(6) Wesson, J, “Tokamaks (Second edition)”, The Oxford Engineering Series 48, Clarendon Press, Oxford (1997).

(7) Lao, L.L., St. John, H., Stambaugh, R.D., Kellman, A.G., Pfeiffer, W., Nuclear Fusion, 25 (1985) 1611.

(8) Lao, L.L., Ferron, J.R., Geoebner, R.J., Howl, W., St. John, H.E., Strait E.J., Taylor, T.S., Nuclear Fusion, 30 (1990) 1035.

(9) Itagaki, M., Kamisawada, J., Oikawa, S., Nuclear Fusion, 44 (2004) 427.

(10) Braams, B.J., Interpretation of tokamak magnetic diagnostics, report IPP 5/2, Max Planck Institut fur Plasma Physik (1985).

(11) Hakkarainen, S.P., Freidberg, J.P., Reconstruction Of Vacuum Flux Surfaces From Diagnostic Measurements In A Tokamak, report PFC/RR-87-22, MIT Plasma Fusion Center (1987). 
(12) Takeda, T., Tokuda, S., Computation of MHD equilibrium of tokamak plasma, Journal of Computational Physics, 93[1] (1991) 1-107.

(13) Press, W.H., Flannery, B.P., Teukolsky, S.A., Vetterling, W.T., “Numerical Recipes - The Art of Scientific Computing”, Cambridge University Press, Cambridge (1986).

(14) Kurihara, K., Fusion Technology, 34 (1998) 548.

(15) Pustovitov , V.D., Nuclear Fusion, 41[6] (2001) 721.

(16) Braams,B.J., Plasma Phys. Contol. Fusion, 33 (1991) 715

(17) Batha, S.H., Levinton, F.M. et al., Nuclear Fusion, 36 (1996) 1133.

(18) Petty, C.C., Fox, W.R., Luce, T.C., Makowski, M.A., Suzuki, T., Nuclear Fusion, 42 (2002) 1124.

(19) Hansen, P.C., “Rank-Deficient and Discrete Ill-Posed Problems - Numerical Aspects of Linear Inversion”, SIAM, Philadelphia (1998).

(20) Azumi, M., Kurita, G., Matsuura, T. et al., in "Computing Methods in Applied Science and Engineering”, p.335, North-Holland, Amsterdam / New York / Oxford (1980).

(21) Brebbia, C.A., “The Boundary Element Method for Engineers”, Pentech Press, London (1978).

(22) Itagaki, M., J. Nucl. Sci. Tech., 22[6] (1985) 565.

(23) Itagaki, M., Engineering Analysis, 4[4] (1987) 190. 


\section{List of Figures}

Figure 1. Illustration of Cauchy-condition Surface Method

Figure 2. Twenty-eight pairs of equilibrium conditions on a magnetic surface

Figure 3. Plasma boundary shape reconstructed using the CCS method

Figure 4. Comparison of boundary values of poloidal field calculated using the CCS method with the direct BEM solutions

Figure 5. Reconstructed results for a parabolic current profile (the initial stage; $\gamma=10^{-9}$ )

Figure 6. Reconstructed results for a parabolic current profile (the 5th iteration; $\gamma=10^{-9}$ )

Figure 7. Reconstructed current profiles with noise effects (the 5th iteration; $\gamma=10^{-9}$ )

Figure 8. Reconstructed results for a hollow current profile (the 5th iteration; $\gamma=10^{-8}$ )

Figure 9. Circular current profiles reconstructed in the 10th iterations 


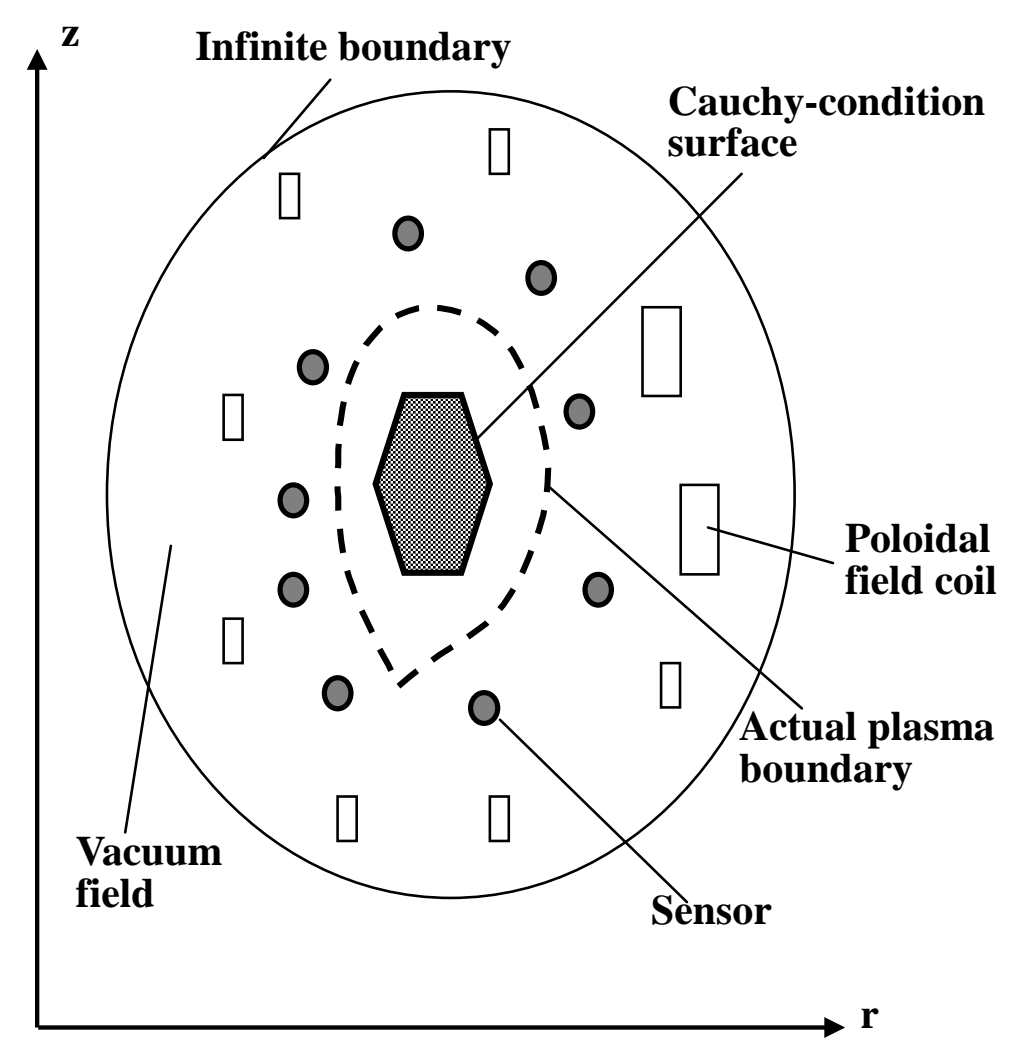

Figure 1. Illustration of Cauchy-condition surface method 


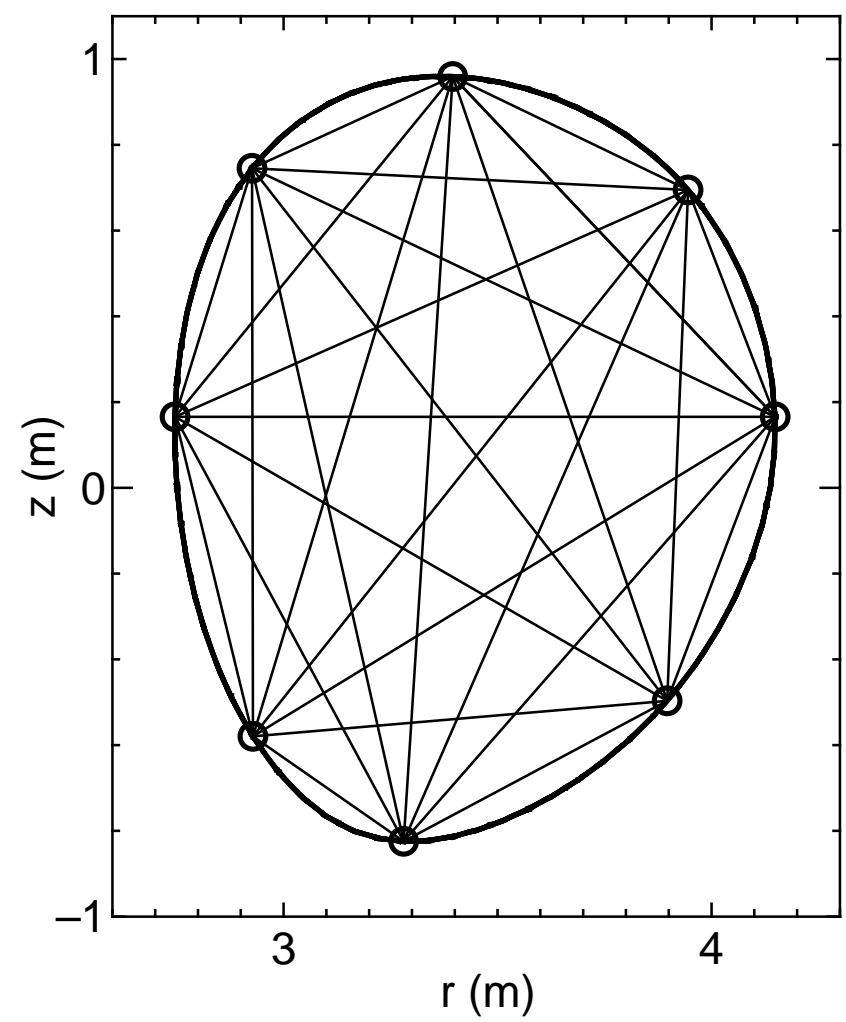

Figure 2. Twenty-eight pairs of equilibrium conditions on a magnetic surface 


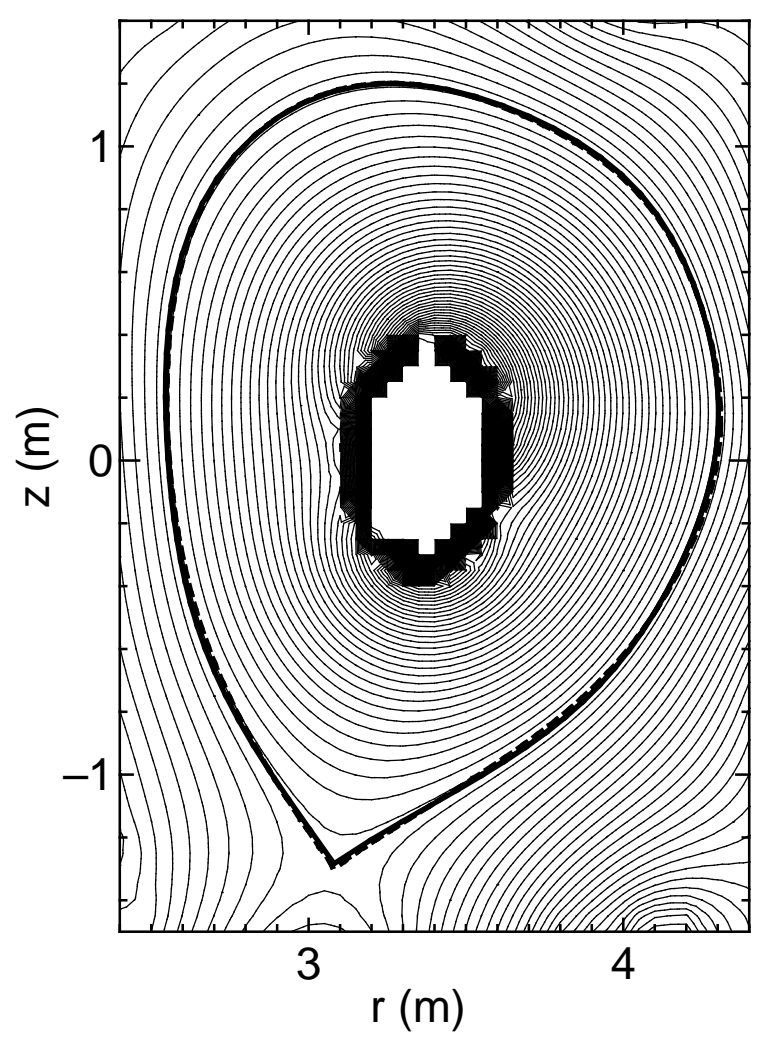

Figure 3. Plasma boundary shape reconstructed using the CCS method 


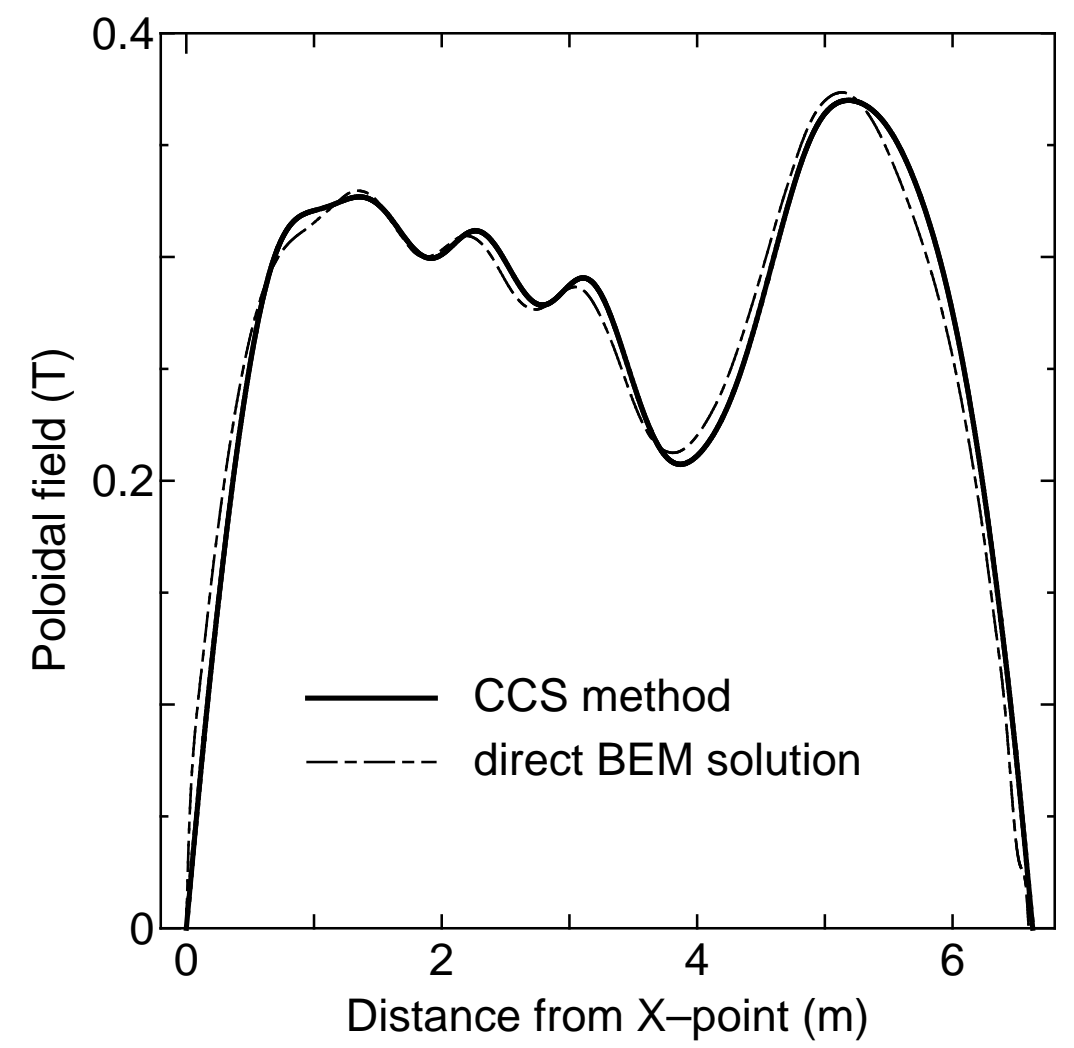

Figure 4. Comparison of boundary values of poloidal field calculated using the CCS method with the direct BEM solutions 


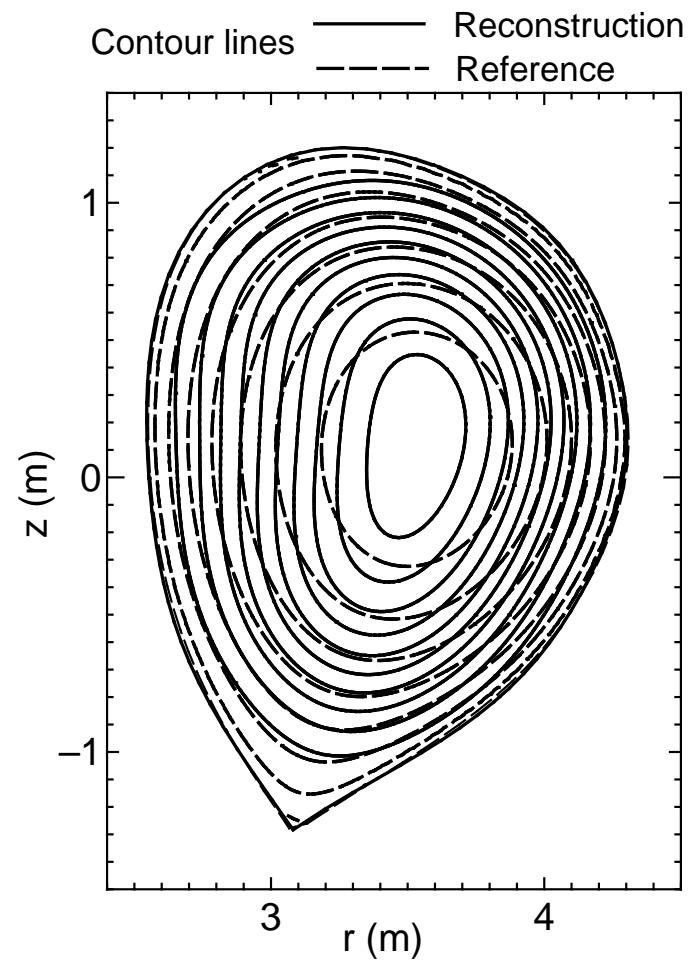

(a) Current density profile

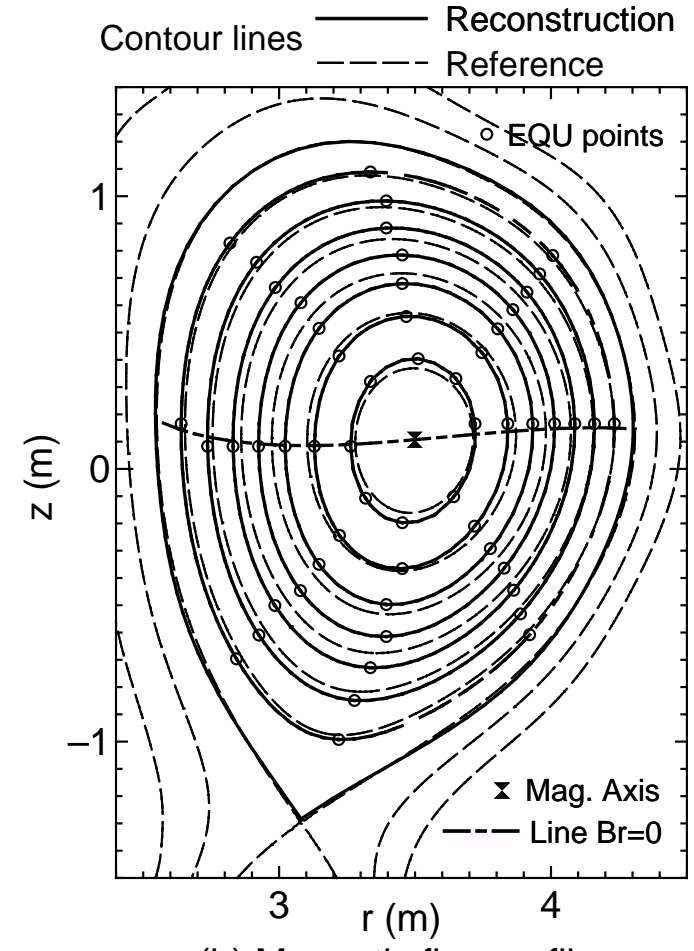

(b) Magnetic flux profile

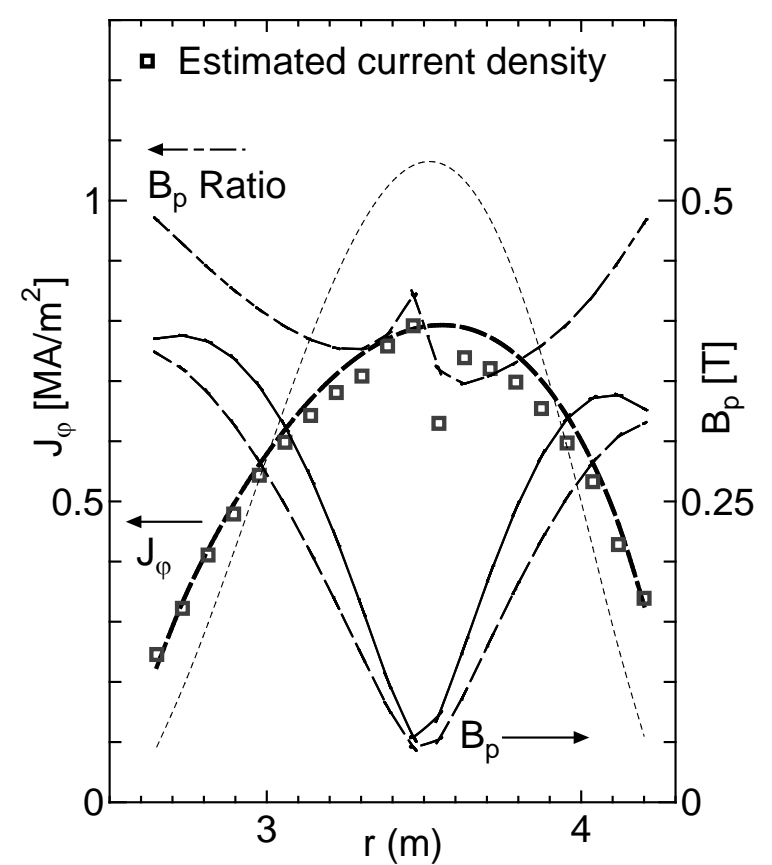

(c) Current density estimated from measured poloidal field

Figure 5. Reconstructed results for a parabolic current profile (the initial stage; $\gamma=10^{-9}$ ) 


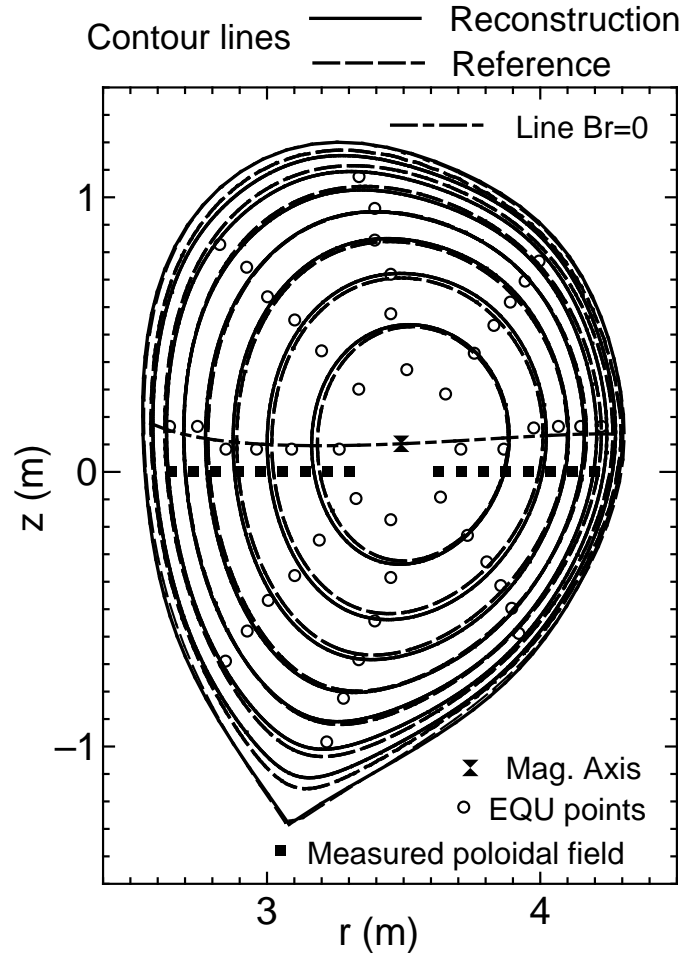

(a) Current density profile

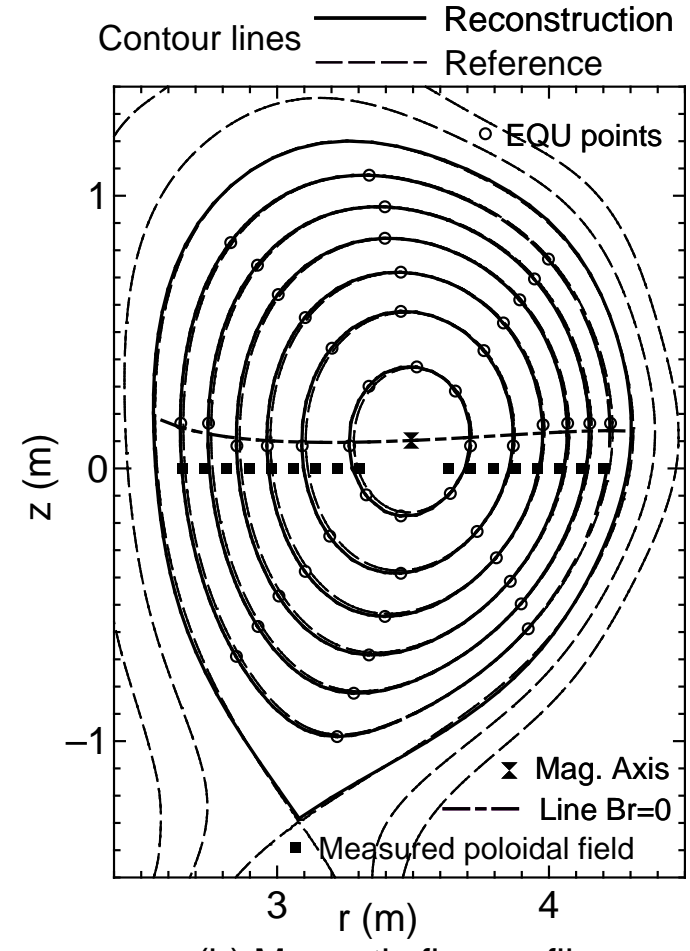

(b) Magnetic flux profile

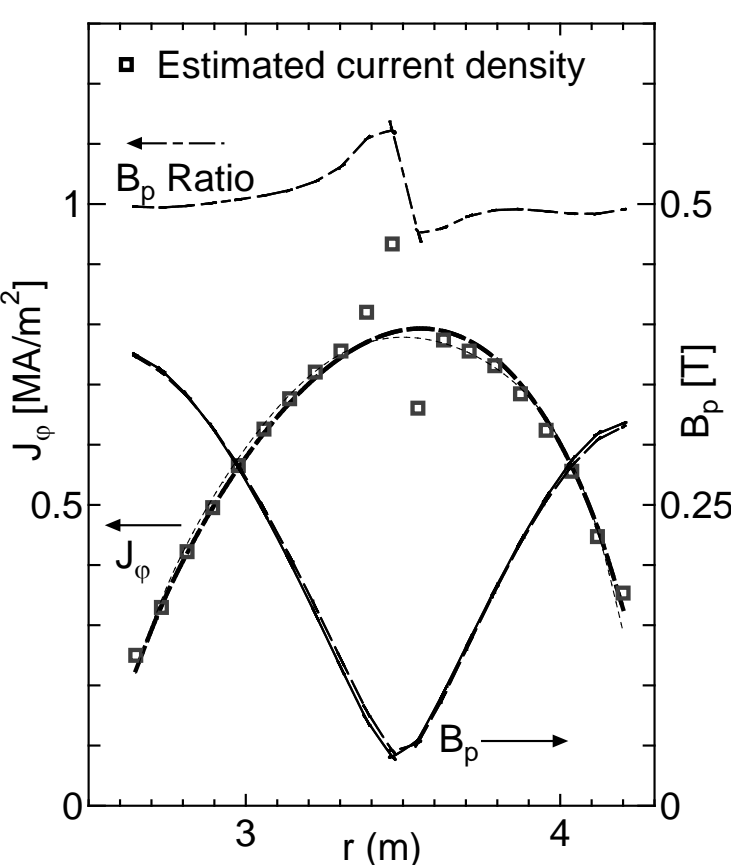

(c) Current density estimated from measured poloidal field

Figure 6. Reconstructed results for a parabolic current profile (the 5th iteration; $\gamma=10^{-9}$ ) 


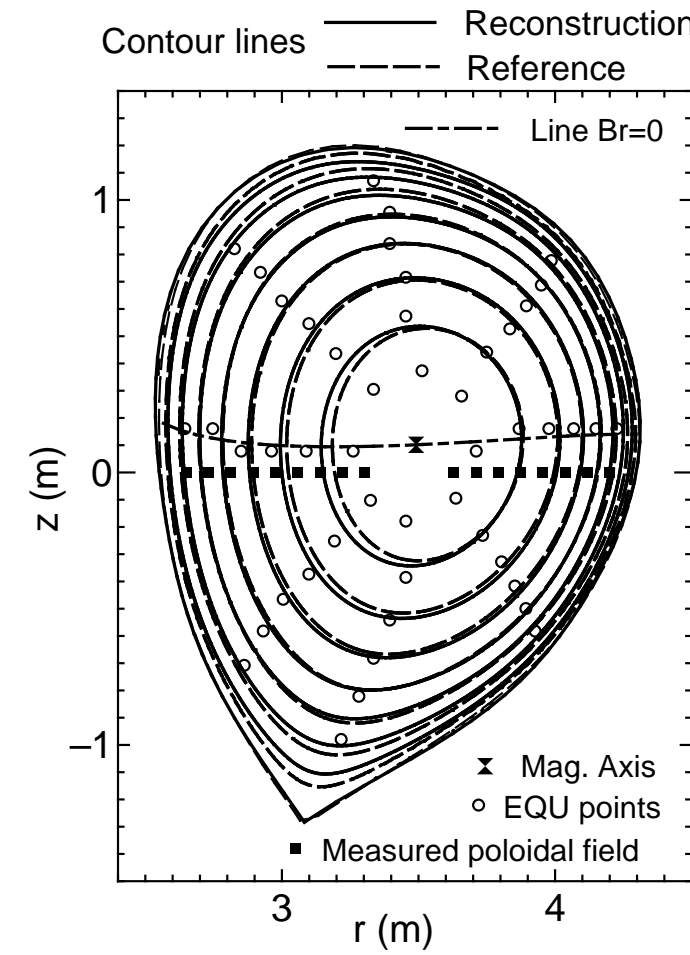

(a) Noised poloidal field data with noised magnetic sensor signals

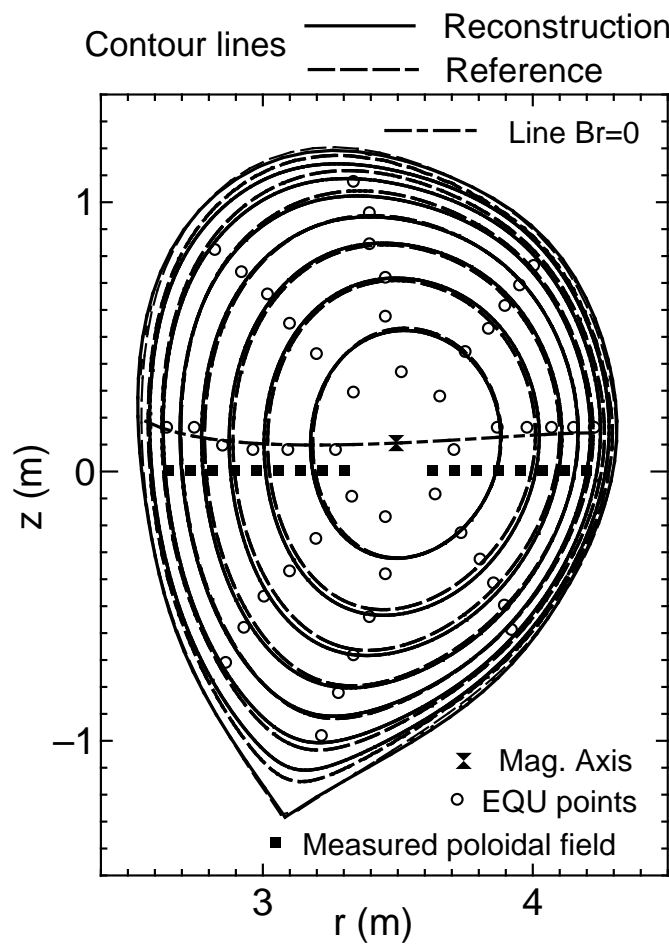

(b) Smoothed polodal field data with noised magnetic sensor signals

Figure 7. Reconstructed current profiles with noise effects (the 5th iteration; $\gamma=10^{-9}$ ) 


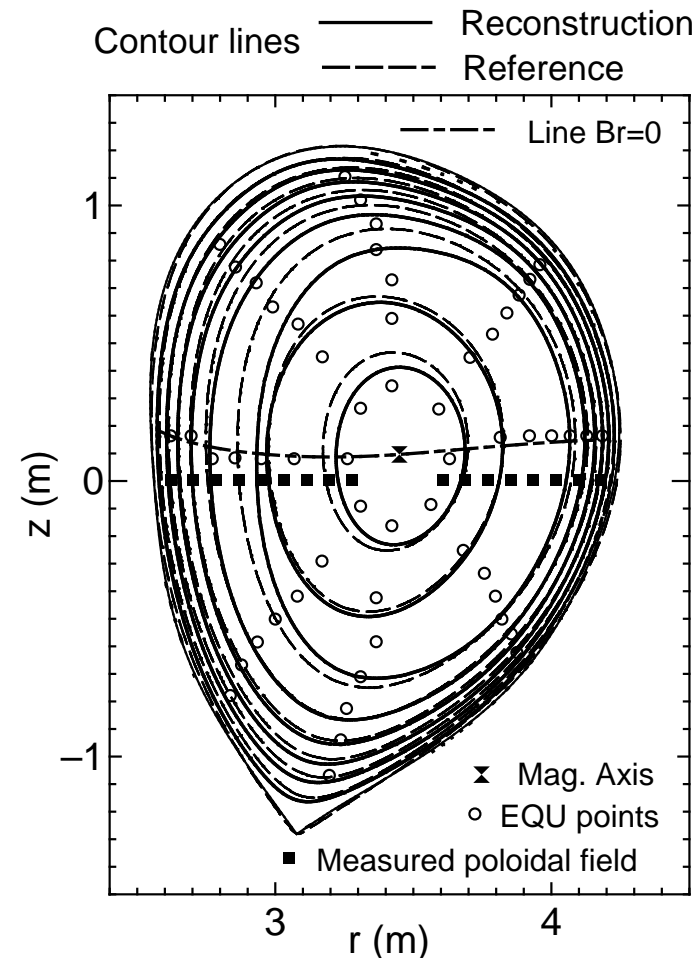

(a) Current density profile

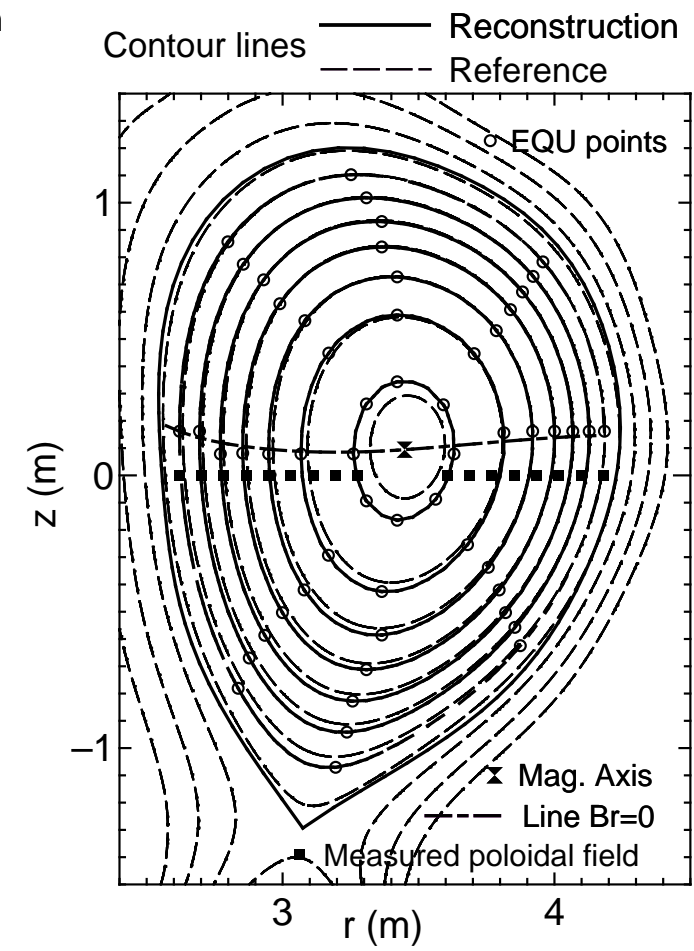

(b) Magnetic flux profile

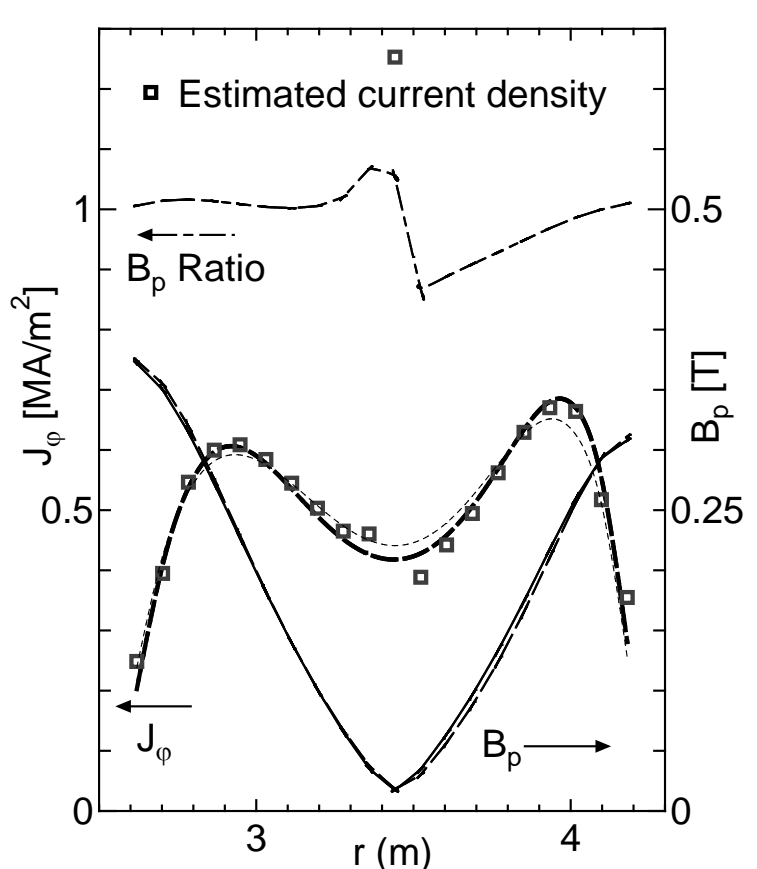

(c) Current density estimated from measured poloidal field

Figure 8. Reconstructed results for a hollow current profile (the 5th iteration; $\gamma=10^{-8}$ ) 


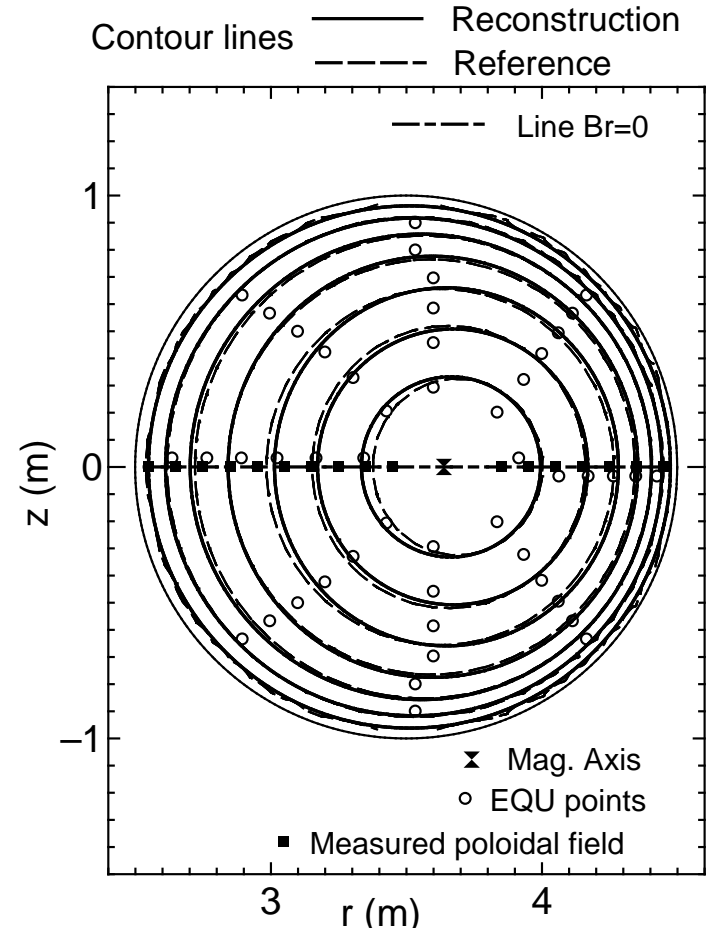

(a) $\gamma=10^{-9}$; Obtained through the same process shown in section 5

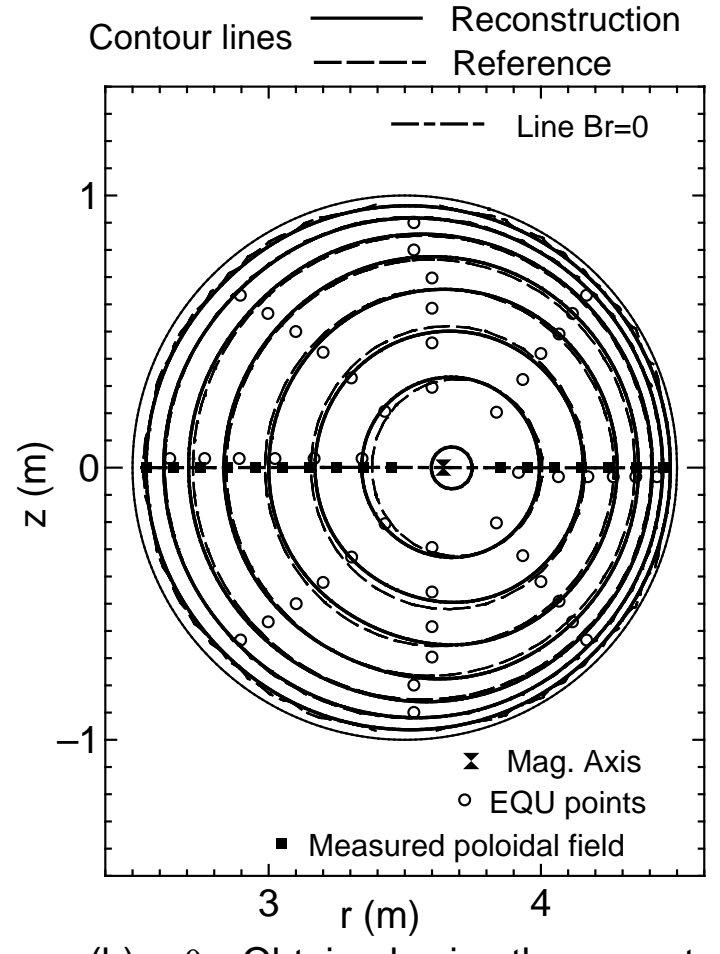

(b) $\gamma=0$. ; Obtained using the current up-down symmetry option

Figure 9. Circular current profiles reconstructed in the 10th iterations 